\title{
Co-evolution of bulges and black holes
}

\author{
Jong-Hak Woo ${ }^{1}$, Tommaso Treu ${ }^{1}$, Matthew A. Malkan ${ }^{2}$ and \\ Roger D. Blandford ${ }^{3}$ \\ ${ }^{1}$ Department of Physics, University of California, Santa Barbara, CA 93106, USA \\ email: woo@physics.ucsb.edu,tt@physics.ucsb.edu \\ ${ }^{2}$ Department of Physics and Astronomy, University of California at Los Angeles, CA \\ 90095-1547, USA email: malkan@astro.ucla.edu \\ ${ }^{3}$ Kavli Institute for Particle Astrophysics and Cosmology, Stanford, CA 94305, USA \\ email: rdb@slac.stanford.edu
}

\begin{abstract}
In the present-day universe, the global properties of bulges and early-type galaxies correlate with the mass of their central black holes, indicating a connection between galaxy evolution and nuclear activity. Understanding the origin of this relation is a major challenge for cosmological models. Using Keck spectra and HST images, we present direct measurements of the correlations between black hole mass and host spheroid luminosity and velocity dispersion at $\mathrm{z}=0.36$, showing that the relations evolved significantly in the past 4 billion years. It appears that black holes of a few $10^{8} \mathrm{M}_{\odot}$ completed their growth before their host galaxies, and that the current scaling relations are only the final point of the co-evolution of galaxies and black holes.
\end{abstract}

Keywords. black hole physics, galaxies: evolution

\section{Introduction}

In the local Universe, the mass of the black holes $\left(\mathrm{M}_{\mathrm{BH}}\right)$ correlates with global properties of their host galaxies, such as the stellar velocity dispersion $(\sigma)$ and luminosity of the spheroidal component $\left(\mathrm{L}_{\text {bulge }}\right)$, indicating a connection between galaxy formation and AGN activity (Ferrarese \& Merritt 2000; Gebhardt et al. 2000). Understanding the origin of these scaling relations is a major challenge for cosmological models and is believed to hold a key to solving several astrophysical problems such as the role of feedback from nuclear activity in suppressing star formation in massive galaxies (e.g. Di Matteo et al. 2005; Croton et al. 2006; Ciotti \& Ostiker 2007).

In the standard cosmological model, spheroids grow by mergers of smaller galaxies while black holes grow by accreting surrounding matter. Depending on the relative timing of the two processes, the scaling relations between $\mathrm{M}_{\mathrm{BH}}$ and velocity dispersion or spheroid luminosity could also evolve with cosmic time. (e.g. Kauffmann \& Haenhelt 2000; Volonteri et al. 2003; Croton 2006). One of the most powerful observational tests of the proposed scenarios is to measure the evolution of the scaling relations with redshift. In this study, we investigate the evolution of the scaling relations, using a carefully selected sample of Seyfert galaxies at $z=0.36 \pm 0.01$.

\section{Sample Selection}

From the SDSS archive, we selected a sample of 22 low-luminosity Seyfert 1 galaxies, where the fraction of stellar light is large enough to measure stellar velocity dispersion. At the same time, virial $\mathrm{M}_{\mathrm{BH}}$ can be obtained from the integrated properties of the broad line region (BLR) using the same spectrum. To minimize the systematic uncertainties related to sky subtraction and atmospheric absorption corrections, we chose specific 
redshift windows where the relevant emission and absorption lines $(\mathrm{H} \beta, \mathrm{Mgb}$, and $\mathrm{Fe}$ ) fall in clean regions of the atmosphere. As a first step, we chose a redshift window at $z=0.36 \pm 0.01$, with a corresponding look-back time of $\sim 4$ Gyrs.

\section{Measurements}

\subsection{Stellar Velocity Dispersion, Bulge Luminosity, and Black Hole Mass}

With high S/N Keck spectra, we measured $\sigma$ for 14 objects, by comparing the observed host galaxy spectra with stellar templates broadened with a Gaussian velocity in spectral regions around Mgb $5175 \AA$ and Fe $5270 \AA$ (see Woo et al. 2006).

Two-dimensional surface photometry was performed with the HST ACS data using the GALFIT software (Peng et al. 2002). We used point source, bulge, and disk components for fitting (see Treu et al. 2007). If needed, an additional component with a Sersic profile with $\mathrm{n}=0.5$ was added to model a bar. L bulge was measured for 17 objects (including 5 upper limits) while 3 objects were excluded due to the presence of dust lanes. We corrected $\mathrm{L}_{\text {bulge }}$ for the passive evolution due to the aging of stellar populations, in order to compare our sample with the local samples.

$\mathrm{M}_{\mathrm{BH}}$ was estimated using the 'virial' method, assuming that the kinematics of the BLR trace the gravitational field of the central black hole (Wandel et al. 1999; Kaspi et al. 2005; Bentz et al. 2006; McGill et al. 2007). The virial coefficient is obtained by requiring that local AGN hosts and quiescent galaxies obey the same $\mathrm{M}_{\mathrm{BH}}-\sigma$ relation (Onken et al. 2004). In practice, $\mathrm{M}_{\mathrm{BH}}$ was estimated using the following equation:

$$
\log M_{\mathrm{BH}}=8.58+2 \log \left(\frac{\sigma_{\mathrm{H} \beta}}{3000 \mathrm{kms}^{-1}}\right)+0.518 \log \left(\frac{L_{5100}}{10^{44} \mathrm{ergs}^{-1}}\right),
$$

where $\sigma_{\mathrm{H} \beta}$ is the second moment of the $\mathrm{H} \beta$ line profile and $L_{5100}$ is the nuclear luminosity at $5100 \AA$ measured from our HST analysis.

\subsection{Local Comparison Samples}

To study evolutionary effects we consider two comparison samples to define the local scaling relations. The first sample is composed of quiescent galaxies with $\mathrm{M}_{\mathrm{BH}}$ determined from spatially resolved kinematics (Marconi \& Hunt 2003). The second sample is local Seyfert galaxies with reverberation $\mathrm{M}_{\mathrm{BH}}$ (Peterson et al. 2004). To ensure self-consistenty we measured the parameters of the local sample in exactly the same way as we do for distant galaxies, using SDSS images, that have intrinsic resolution similar to that of HST-ACS images of our distant galaxies (approximately 10 times larger PSF, but 10 times smaller angular distance).

The first sample is larger in size with robustly measured $\mathrm{M}_{\mathrm{BH}}$ and spheroid luminosities. However, the local Seyferts are more appropriate for a direct comparison: i) although the virial coefficient of the BLR is unknown, the comparison between Seyfert samples is independent of its numerical value; ii) if the local scaling relation is not universal, Seyferts may define a different $\mathrm{M}_{\mathrm{BH}}-\mathrm{L}_{\text {bulge }}$ relation than quiescent galaxies. Thus, the more conservative approach is to consider both samples for the evolution analysis.

\section{Scaling Relations at $z=0.36$}

Figure 2 shows the scaling relations of the $\mathrm{z}=0.36$ sample, compared with the local samples. The $\mathrm{M}_{\mathrm{BH}}-\sigma$ relation shows that at fixed host galaxy velocity dispersion, distant Seyferts appear to host larger $\mathrm{M}_{\mathrm{BH}}$ than local ones. The average offset corresponds to $\Delta \log \sigma=0.13 \pm 0.03 \pm 0.05$ (i.e. $\Delta \log M_{\mathrm{BH}}=0.54 \pm 0.12 \pm 0.21$ ). 


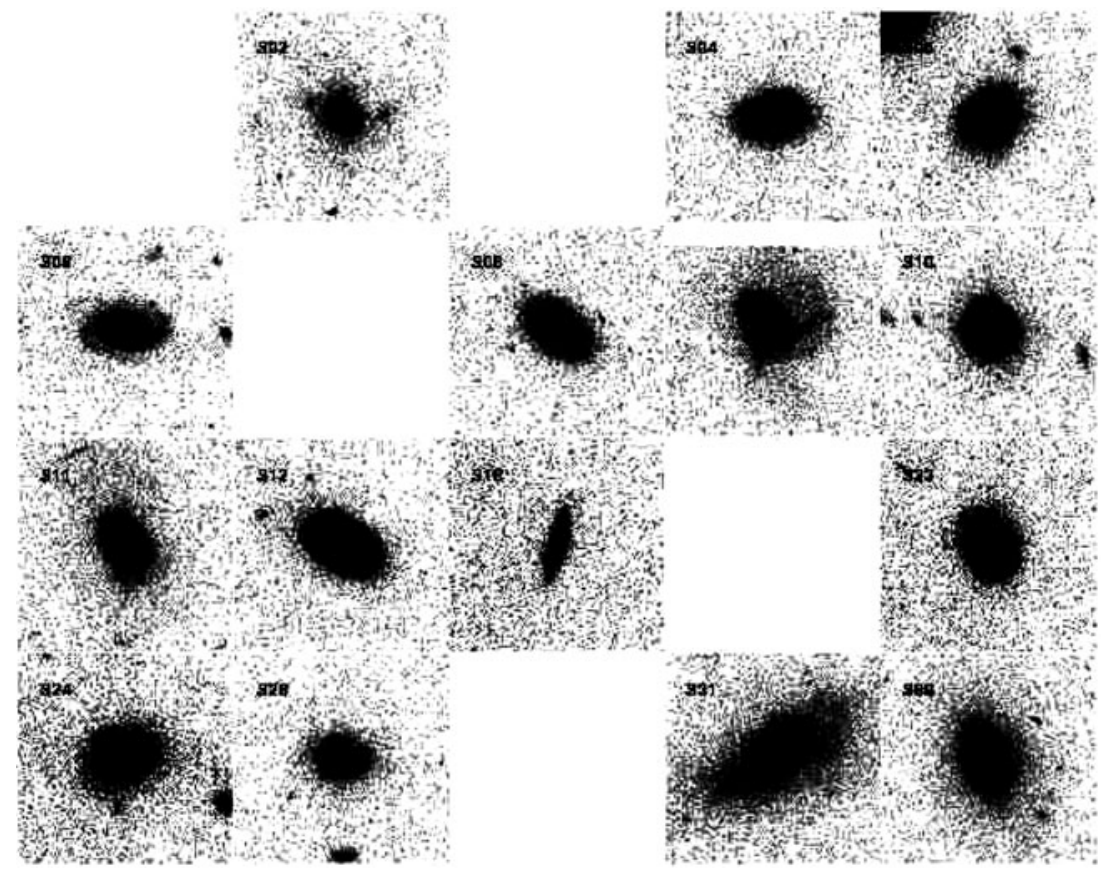

Figure 1. HST images of the 20 Seyfert galaxies in the sample. Each postage stamp is 12 arcseconds on a side, i.e. approximately $60 \mathrm{kpc}$. Six objects show merging or interaction, denoted by the label $\mathrm{M}$ or $\mathrm{M} / \mathrm{I}$.
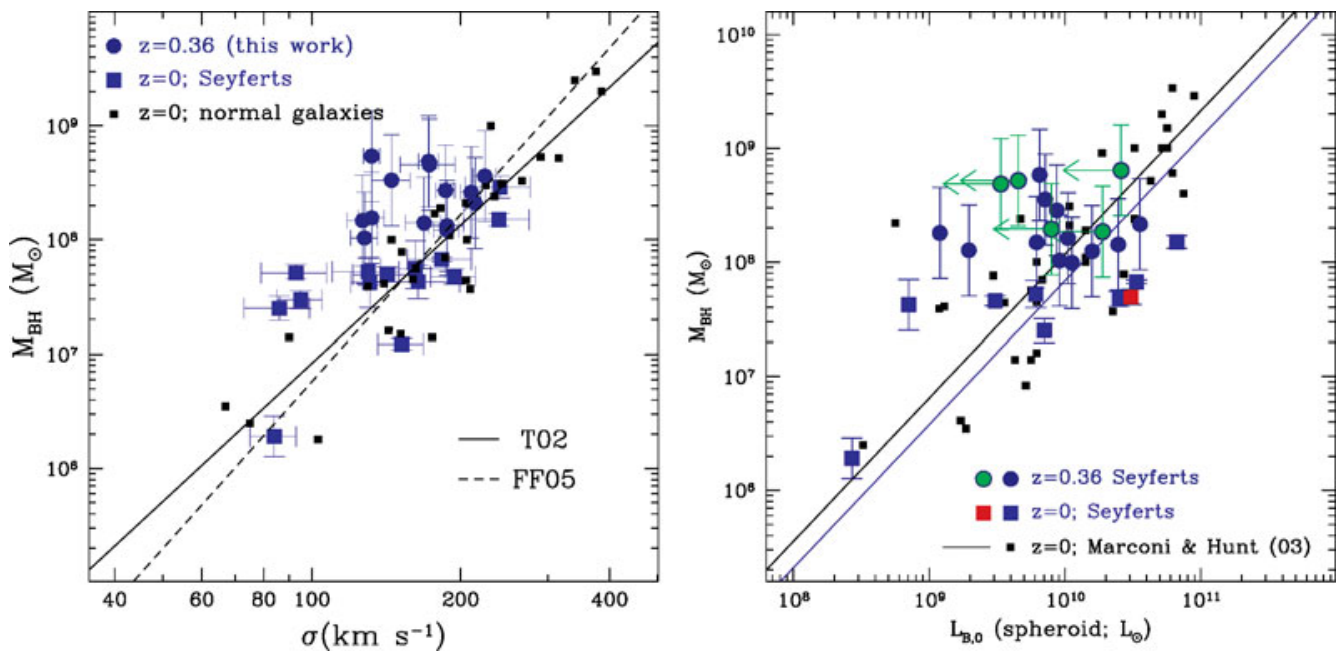

Figure 2. Left: The $\mathbf{M}_{\mathrm{BH}}-\sigma$ relation for distant Seyferts (blue circles), local Seyferts (magenta squares), and local quiescent galaxies (black squares), compared with the best fit relations from Tremaine et al. 2002 (solid line) and Ferrarese \& Ford 2005 (dashed line). Right: The $\mathbf{M}_{\mathrm{BH}}-\mathbf{L}_{\mathrm{bulge}}$ relation for distant Seyferts (large circles; blue: measurements; cyan: upper limits to $\mathrm{L}_{\mathrm{bulge}}$ ). Local Seyferts are shown as large squares (magenta: measurements; red: an upper limit to $\mathrm{L}_{\mathrm{bulge}}$ ). Black squares represent local quiescent galaxies from Marconi \& Hunt (2003). The black line is the best fits to the Marconi \& Hunt sample. The magenta line is the best fit to the local Seyferts. For direct comparison Lbulge of the distant Seyferts has been passively evolved to $z=0$ using $\log \mathrm{L}_{\mathrm{B}, 0}=\log L_{\mathrm{B}, \mathrm{z}}-0.72 z$. 
The $\mathrm{M}_{\mathrm{BH}}-\mathrm{L}_{\mathrm{bulge}}$ relation shows the same basic result. The Seyfert galaxies at $z=0.36$ and those at $z=0$ cover approximately the same range in spheroid luminosity, but the average $\mathrm{M}_{\mathrm{BH}}$ is higher for the distant Seyferts. The average offset with respect to the two local samples corresponds to $\Delta L_{\mathrm{B}, 0}=0.40 \pm 0.11 \pm 0.15\left(\Delta \log M_{\mathrm{BH}}=0.51 \pm 0.14 \pm\right.$ 0.19). The observed offset is a lower limit considering that five of the distant Seyferts measurements are upper limits (while only one of the local Seyferts is an upper limit).

\section{Discussion and Conclusions}

The two scaling relations show a significant offset from the local relationship. To satisfy the local $\mathrm{M}_{\mathrm{BH}}-\sigma$ and $\mathrm{M}_{\mathrm{BH}}-\mathrm{L}$ relations by $z=0$ - assuming no black hole growth - our distant spheroids have to grow their stellar mass by approximately $60 \%$ in the next 4 billion years, while preserving their size and stellar mass-to-light ratio approximately constant. This corresponds to an evolution of the black hole to spheroid mass ratio of the form $M_{\mathrm{BH}} / M_{\mathrm{sph}} \propto(1+z)^{1.5 \pm 1.0}$.

Selection bias may be present in our sample (selected against low luminosity AGNs and thus small $\mathrm{M}_{\mathrm{BH}}$ ), possibly resulting in the observed evolution of the scaling relations. However, our Monte Carlo simulation study indicates that only 0.1 dex offset could be resulted from the selection effect (see also Lauer et al. 2007 for similar results), suggesting that selection bias cannot entirely account for the offset. Larger samples of AGNs with determined $\mathrm{M}_{\mathrm{BH}}, \sigma$, and host galaxy properties are needed both locally and at highredshift to improve the understanding of selection effects.

The observed evolution can be qualitatively explained if our Seyferts undergo a single collisional merger with a disk-dominated system between $\mathrm{z}=0.36$ and today. A single merger could increase the spheroid mass by transporting stellar mass from the progenitors disks. At the same time, this process would add younger stars to the spheroid (either from the merging disks or from newly formed stars) thus counteracting the fading of the old stellar populations and producing an approximately constant stellar mass-to-light ratio in the spheroid. Numerical simulations including realistic prescriptions for star formation, AGN activity and mass loss will be needed to see if these mergers preserve $\mathrm{R}_{\mathrm{e}}$ and $\mathrm{M}_{\mathrm{sph}} / \mathrm{L}_{\mathrm{B}}$, which will confirm that black holes completed their growth before their host galaxies at these mass scales $\left.(<\sigma\rangle=170 \mathrm{~km} \mathrm{~s}^{-1}\right)$.

\section{References}

Bentz, M. C., et al. 2006, ApJ, 644, 133

Ciotti, L. \& Ostriker, J. P. 2007, ApJ, 665, 1038

Croton, D. J. 2006, MNRAS, 369, 1808

Di Matteo, T., et al. 2005, Nature, 433, 604

Ferrarese, L. \& Merritt, D. 2000, ApJL, 539, L9

Gebhardt, K., et al. 2000, ApJL, 539, L13

Kaspi, S., et al. 2005, ApJ, 629, 61

Kauffmann, G., \& Haehnelt, M. 2000, MNRAS, 311, 576

Marconi \& Hunt 2003, ApJ, 589, L1

McGill, K., Woo, J.-H., Treu, T., \& Malkan, M. 2007, ApJ, submitted

Onken, C. A., et al. ApJ, 615, 645

Peng, C. et al. 2002, AJ, 124, 266

Tremaine, S., et al. 2002, ApJ, 574, 740

Volonteri, Haardt \& Madau 2003, ApJ, 582, 559

Woo, J.-H., Treu, T, Malkan, M. A., \& Blandford, R. D. 2006, ApJ, 645, 900

Treu, T., Woo, J.-H., Malkan, M. A., \& Blandford, R. D. 2007, ApJ, 667, 117 